\title{
Cost-utility analysis of treatment options after initial tumor necrosis factor inhibitor therapy discontinuation in patients with rheumatoid arthritis
}

\author{
Aliza R Karpes Matusevich, PhD; Lincy S Lal, PhD; Wenyaw Chan, PhD; J Michael Swint, PhD; Scott B Cantor, PhD; \\ Maria E Suarez-Almazor, MD, PhD; and Maria A Lopez-Olivo, MD, PhD
}

\section{What is already known about this subject}

- For patients with rheumatoid arthritis (RA) who have not responded to their first tumor necrosis factor inhibitor (TNFi), there are 2 basic approaches to treatment: cycling (switching to another TNFi) or switching to a drug with another mechanism of action.

- A published report from an administrative claims database showed that adalimumab (another TNFi) and abatacept (selective T-cell costimulation blocker) were more commonly prescribed after first TNFi discontinuation in the United States.

\section{What this study adds}

- The cost-utility of adalimumab versus abatacept was evaluated in patients with RA whose initial TNFi therapy failed.

- Study results showed that switching to abatacept was a cost-effective strategy for RA patients who discontinue initial therapy with TNFi at a willingness-topay threshold of $\$ 100,000$ per QALY.

\author{
Author affiliations \\ Aliza R Karpes Matusevich, PhD, and \\ Lincy S Lal, PhD, Department of \\ Management, Policy and Community \\ Health, School of Public Health, The \\ University of Texas Health Science Center at \\ Houston. Wenyaw Chan, PhD, Department \\ of Biostatistics and Data Science, School \\ of Public Health, and J Michael Swint, \\ PhD, Department of Management, Policy \\ and Community Health, School of Public \\ Health, and Center for Clinical Research and \\ Evidence-Based Medicine, McGovern School \\ of Medicine, The University of Texas Health \\ Science Center at Houston. Scott B Cantor, \\ $\mathrm{PhD}$, and Maria A Lopez-Olivo, MD, PhD, \\ Department of Health Services Research, \\ The University of Texas MD Anderson Cancer \\ Center, Houston. Maria E Suarez-Almazor, \\ MD, PhD, Department of Health Services \\ Research and Section of Rheumatology and \\ Clinical Immunology, Department of General \\ Internal Medicine, The University of Texas \\ MD Anderson Cancer Center, Houston. \\ AUTHOR CORRESPONDENCE: \\ Maria A Lopez-Olivo, 713.792.9175; \\ amlopezo@mdanderson.org
}




\section{ABSTRACT}

BACKGROUND: For patients with rheumatoid arthritis (RA) who discontinued initial treatment with tumor necrosis factor inhibitor (TNFi), 2 approaches are commonly used: cycling to another TNFi or switching to a drug with another mechanism of action. Currently, there is no consensus on which approach to use first. A report from the IBM MarketScan Research administrative claims database showed adalimumab (cycling strategy) and abatacept (switching strategy) were more commonly prescribed after the first TNFi discontinuation.

OBJECTIVE: To evaluate the cost-utility of adalimumab versus abatacept in patients with RA whose initial TNFi therapy failed.

METHODS: A probabilistic cost-utility microsimulation state-transition model was used. Our target population was commercially insured adults with RA, the time horizon was 10 years, and we used a payer perspective. Patients not responding to adalimumab or abatacept were moved to the next drug in a sequence of 3 and, finally, to conventional synthetic therapy. Incremental cost-utility ratios (2016 USD per quality-adjusted-life-year gained [QALY)] were calculated. Utilities were derived from a formula based on the Health Assessment Questionnaire Disability Index and age-adjusted comorbidity score.

RESULTS: Switching to abatacept after the first TNFi showed an incremental cost of just more than $\$ 11,300$ over 10 years and achieved a QALY benefit of 0.16 compared with adalimumab. The incremental cost-effectiveness ratio was $\$ 68,950$ per QALY. Scenario analysis produced an incremental cost-effectiveness ratio range of $\$ 44,573$ per QALY to $\$ 148,558$ per QALY. Probabilistic sensitivity analysis showed that switching to abatacept after TNFi therapy failure had an $80.6 \%$ likelihood of being cost-effective at a willingness-to-pay threshold of $\$ 100,000$ per QALY.

CONCLUSIONS: Switching to abatacept is a cost-effective strategy for patients with RA whose discontinue initial therapy with TNFi.

Rheumatoid arthritis (RA) is a chronic inflammatory disease of the joints affecting more than 1.3 million Americans. Meta-analyses have estimated the annual direct medical costs associated with RA as $\$ 12,509$ for all patients and more than $\$ 36,000$ for patients using biological diseasemodifying antirheumatic drugs (DMARD). ${ }^{1}$ Therapy with tumor necrosis factor inhibitors (TNFi) greatly improved the management of patients with RA by suppressing the inflammatory effects of TNF alpha. However, evidence from clinical trials and claims data suggests that between $30 \%$ and $>60 \%$ of patients do not experience an adequate response to these drugs, necessitating a change in treatment regimen. ${ }^{2-5}$

There are 2 basic approaches to treatment: cycling (switching to another TNFi) or switching to a drug with another mechanism of action. These non-TNFi drugs work by blocking the inflammatory effects of cytokines such as interleukin-6 (tocilizumab, sarilumab); depleting B cells (rituximab); or inhibiting $\mathrm{T}$ cells (abatacept) or the Janus kinase (JAK) enzymes (tofacitinib, baracitinib, peficitinib, upadacitinib). The optimal choice is unclear. Neither the American College of Rheumatology nor the European League Against Rheumatism guidelines provide definitive guidance. ${ }^{6,7}$

A previous study showed that most (63.5\%) commercially insured patients cycle to another TNFi. Of these, a plurality cycle to adalimumab, and of the patients who switch to non-TNFi drugs, most switch to abatacept. ${ }^{8}$ Results from effectiveness studies are mixed, seeming to indicate greater clinical improvement with non-TNFi drugs. ${ }^{9-16}$ In addition to questions about efficacy, few cost-effectiveness models have considered options after TNFi failure, ${ }^{17}$ and we did not find a full model based on a real-world U.S. population.

In this study, we performed an economic evaluation of alternative treatments for adult RA patients in the United States whose initial TNFi therapy failed based on data from real-world practice.

\section{Methods}

We followed guidelines by Philips et al. (2006) for good practice in decision-analytic modeling ${ }^{18}$ and based our model structure on best practices as set out in the reference case recommendations made by the U.S. Panels on CostEffectiveness in Health and Medicine. ${ }^{19}$ These guidelines are, by design, broad; therefore, RA-specific methodology followed the Outcome Measures in Rheumatology Clinical Trials (OMERACT) consensus-based reference case for $R A,{ }^{20,21}$ with input from a systematic review of the RA costeffectiveness literature. ${ }^{17}$ We report our methods according to the Consolidated Health Economic Evaluation Reporting Standards statement. ${ }^{22}$ This study used deidentified and aggregated data already published in the literature. It did not involve human participants and as such did not require approval from an institutional review board.

\section{TARGET POPULATION AND SETTING}

We created a hypothetical cohort of 10,000 individuals with RA, whose sex, age, and comorbidity status were derived from our previous analysis of adults aged 18 and older with RA who had switched from a TNFi to a biologic (bDMARD) or JAK-inhibitor between 2008 and 2016 in a large U.S. administrative claims database (IBM MarketScan Research). ${ }^{8}$ Baseline Health Assessment Questionnaire Disability 


\section{TABLE 1}

Per Cycle Transition Probabilities of Drug Continuation in the Cycling (Adalimumab-Abatacept-Tocilizumab) and Switching (Abatacept-TocilizumabRituximab) Strategies ${ }^{a}$

\begin{tabular}{|c|c|c|c|c|}
\hline \multirow{2}{*}{$\begin{array}{l}\text { Beta } \\
\text { Distribution }\end{array}$} & Second Drug & Third Drug & \multicolumn{2}{|c|}{ Fourth Drug } \\
\hline & Mean (SEM) & Mean (SEM) & \multicolumn{2}{|c|}{ Mean (SEM) } \\
\hline \multicolumn{5}{|l|}{ Cycling } \\
\hline Cycle 1 & $0.64 \quad(0.25)$ & $0.69 \quad(0.16)$ & 0.59 & $(0.10)$ \\
\hline Cycle 2 & $0.74 \quad(0.19)$ & $0.59 \quad(0.14)$ & 0.64 & $(0.07)$ \\
\hline Cycle 3 & $0.71 \quad(0.16)$ & $0.67 \quad(0.10)$ & 0.66 & $(0.06)$ \\
\hline Cycle 4 & $0.70 \quad(0.14)$ & $0.74 \quad(0.08)$ & 0.70 & $(0.05)$ \\
\hline Cycle 5 & $0.73 \quad(0.11)$ & $0.73 \quad(0.07)$ & 0.73 & $(0.04)$ \\
\hline Cycle 6 & $0.75 \quad(0.09)$ & $0.68 \quad(0.06)$ & 0.55 & (0.03) \\
\hline Cycle 7 & $0.76 \quad(0.08)$ & $0.77 \quad(0.05)$ & 0.78 & $(0.02)$ \\
\hline Cycle 8+ & $0.70 \quad(0.07)$ & $0.65 \quad(0.05)$ & 0.71 & $(0.02)$ \\
\hline \multicolumn{5}{|l|}{ Switching } \\
\hline Cycle 1 & $0.74 \quad(0.20)$ & $0.65 \quad(0.13)$ & 0.90 & $(0.04)$ \\
\hline Cycle 2 & $0.76 \quad(0.17)$ & $0.62 \quad(0.10)$ & 0.58 & $(0.07)$ \\
\hline Cycle 3 & $0.71 \quad(0.15)$ & $0.69 \quad(0.08)$ & 0.74 & $(0.05)$ \\
\hline Cycle 4 & $0.69 \quad(0.13)$ & $0.63 \quad(0.07)$ & 0.73 & $(0.04)$ \\
\hline Cycle 5 & $0.75 \quad(0.10)$ & $0.69 \quad(0.05)$ & 0.66 & $(0.04)$ \\
\hline Cycle 6 & $0.70 \quad(0.09)$ & $0.71 \quad(0.04)$ & 0.64 & $(0.03)$ \\
\hline Cycle 7 & $0.77 \quad(0.07)$ & $0.60 \quad(0.04)$ & 0.68 & $(0.02)$ \\
\hline Cycle 8+ & $0.70 \quad(0.07)$ & $0.67 \quad(0.03)$ & 0.82 & $(0.02)$ \\
\hline
\end{tabular}

Note: Cycle length is 6 months.

aProbabilities were calculated by dividing the number of patients still receiving the treatment at the end of each 6-month period by the total number of patients continuing follow-up in that period. Rates were assumed to be constant after 4 years, or 8 cycles.

SEM $=$ standard error of the mean

Index (HAQ-DI) values were derived from a computation of patients' age-adjusted comorbidity index, which has been shown to correlate with HAQ-DI. ${ }^{23-26}$ In our dataset, 79.9\% of patients were female, with a mean age (SD) of 52.03 (11.76) years and a calculated mean (SD) HAQ-DI score of $1.46(0.29)$ (Supplementary Table 1, available in online article). Scenario analyses were run using HAQ (SD) values derived from the Birmingham Rheumatoid Arthritis Model (BRAM) ${ }^{27}$ and 2 head-to-head trials comparing cycling versus switching to a non-TNFi drug: the Rotation of anti-TNF Or Change of class of biologic (ROC) $)^{16}$ and Abatacept Trial in Treatment of AntiTNF Inadequate Responders (ATTAIN) ${ }^{28}$ clinical trials, which were $2.0(0.56), 1.3(0.6)$, and $1.8(0.6)$, respectively.

\section{STUDY PERSPECTIVE AND COMPARATORS}

The current economic evaluation was from the perspective of U.S. private health care payers. We included only direct medical costs. ${ }^{19,21,29}$ It is recommended that treatment sequences, rather than individual drugs, are modeled and that these sequences be based on actual practice..$^{19,21}$ The literature on sequences has mostly concentrated on TNFi therapies, and even then, there is no consensus on the most common TNFi used after conventional synthetic (cs) DMARD failure. Many RA patients initiating treatment with TNFi receive etanercept first. ${ }^{8,30-32}$ The most common treatments reportedly prescribed after initial TNFi failure are adalimumab, ${ }^{33-36}$ etanercept, ${ }^{37-39}$ or infliximab..$^{40}$ Only 1 study has examined non-TNFi drugs, reporting that abatacept was used more than $70 \%$ of the time in those switching to a nonTNFi drug. ${ }^{33}$

Comparators for the current study consisted of the most common sequence in each of the cycling and switching to non-TNFI drug categories as ascertained by the analysis of administrative claims data. For cycling, this was adalimumab followed by abatacept and then tocilizumab. ${ }^{8}$ For switching to a non-TNFI drug, this was abatacept followed by tocilizumab and then rituximab. Patients who survived the full treatment sequence moved to csDMARDs after failure of their third drug. Given the paucity of literature on csDMARDs following biological or targeted synthetic DMARDs, we did not specify what therapy was used. ${ }^{41}$

\section{TIME HORIZON AND DISCOUNT RATE}

The model followed patients from initiation of the second bDMARD for 10 years or until death. ${ }^{42,43}$ Theorists prefer a lifetime perspective to reflect the chronic nature of the disease ${ }^{18}$ but for RA specifically, OMERACT cautions against extrapolating beyond available data. ${ }^{21}$ The mean follow-up time for our MarketScan cohort was 2.9 years (SD 1.6, max 8.5). ${ }^{8}$ For the sensitivity analysis, we used lifetime and 5 -year perspectives.

The mortality rate was based on standard U.S. life tables multiplied by a rheumatoid arthritis risk modifier. ${ }^{44,45}$ All costs and outcomes were discounted at a rate of $3 \%$ per annum. ${ }^{19}$

\section{MEASURES OF EFFECTIVENESS, CHOICE OF HEALTH AND PREFERENCE-BASED OUTCOMES}

Initial and continued treatment response (transition) probabilities were determined from the claims data on a semiannual basis. ${ }^{8}$ The model's cycle length of 6 months was chosen based on similar studies and in line with clinical trial outcome reporting. ${ }^{17}$ Transition probabilities were calculated by dividing the number of patients still receiving the treatment at the end of each 6 -month period by the total 


\begin{tabular}{|c|c|c|c|c|c|c|}
\hline TABLE 2 & $\begin{array}{l}\text { Drug } \\
\text { the C } \\
\text { Switc }\end{array}$ & $\begin{array}{l}\text { is for Fi } \\
\text { ig (Ada } \\
\text { (Abata }\end{array}$ & $\begin{array}{l}\text { nd Sul } \\
\text { mab-A } \\
\text {-Tocili }\end{array}$ & $\begin{array}{l}\text { uent } 1 \\
\text { acept- } \\
\text { ab-Rit }\end{array}$ & $\begin{array}{l}\text { ications } \\
\text { lizuma } \\
\text { mab) St }\end{array}$ & $\begin{array}{l}\text { d } \\
\text { gies } \\
\end{array}$ \\
\hline & Cycli & Another & Drug & Switc & to a Non & Drug \\
\hline & Drug & Mean, \$ & SD, $\$$ & Druga & Mean, \$ & SD, \$ \\
\hline First cycle & ADA & 12,873 & 7,277 & ABA & 13,244 & 7,228 \\
\hline & ABA & 13,244 & 7,228 & TCZ & 11,984 & 7,466 \\
\hline & TCZ & 11,984 & 7,465 & RTX & 16,469 & 8,713 \\
\hline Subsequent & ADA & 7,900 & 9,214 & ABA & 7,756 & 7,676 \\
\hline & ABA & 7,756 & 7,676 & TCZ & 8,365 & 8,280 \\
\hline & TCZ & 8,365 & 8,280 & RTX & 8,437 & 8,952 \\
\hline
\end{tabular}

${ }^{a}$ Cost parameters were determined from 2008 to 2016 administrative claims data. Net payments as reported by the insurance carrier were the source for the calculation. Direct drug-related costs comprised acquisition costs for the drugs of interest.

Costs are higher for the first cycle due to increased costs owing to more intensive initial follow-up and, in some cases, loading doses of the drugs.

$A B A=$ abatacept $A D A=$ adalimumab; $R T X=$ rituximab; $T C Z=$ tocilizumab; $T N F i=$ tumor necrosis factor inhibitor.

number of patients continuing followup in that period. Rates were assumed to be constant after 4 years (Table 1 ).

Based on previous cost-effectiveness models, we assumed in our model that responding patients experienced an initial improvement in disability (HAQ-DI reduction) followed by slow disease progression until loss of efficacy (return to baseline HAQ-DI) ${ }^{46-48}$ at which point they moved to the next treatment in sequence. HAQ-DI has been shown to be a close approximation of patients' own evaluation of their health, ${ }^{49}$ with a fundamental relationship to utility and a strong correlation with costs and mortality. ${ }^{45,50}$ We converted HAQ-DI to utilities using the same formula as that of BRAM. ${ }^{27}$ Sensitivity analyses used 2 other formulas: Bansback et al. (2005), ${ }^{51}$ because it is the most frequently used, and Carreño et al. (2011), ${ }^{52}$ because it gives higher values than these and other commonly used formulas.

\section{ESTIMATING RESOURCES AND COSTS}

The current economic evaluation was from the perspective of U.S. private health care payers, as such we included only direct medical costs. . $^{19,21,29}$ Cost parameters and their probability distributions were determined from 2008 to 2016 administrative claims data. Net payments as reported by the insurance carrier were the source for the calculation. We calculated 2 categories of costs: (a) direct drugrelated costs comprising acquisition costs for the drugs of interest and (b) other health care costs consisting of all other claims, such as other drugs, drug administration costs, physician visits, and hospital admissions. Each category was further subdivided into initial cycle or subsequent cycles to account for loading doses, drug administration, and extra monitoring associated with starting a new treatment. The total cost for each cycle was the sum of drug and other health care costs (Table 2). Costs of palliative therapy cycles were assumed to be the same as the other health care costs for non-first cycles, without the addition of drug costs. This included other DMARD therapy patients would have been taking.

Like utilities, and based on a previous report of increased costs with greater disability, ${ }^{53}$ other health care costs were assigned according to each individual's functional disability score in each cycle. ${ }^{54,55}$ The effect of any assumptions made were checked in scenario analyses. In a post hoc subgroup analysis, we explored the costs for 33 individuals aged older than 80 years with 2 or more comorbidities (age-adjusted comorbidity index $=6$ ) and subsequently excluded them from the base-case analysis because these costs were more than double the next highest age-adjusted comorbidity index category and likely included end-of-life costs.

\section{CURRENCY, PRICE DATE, AND CONVERSION}

Costs were adjusted to 2016 USD according to methods specified by the Community Guide using the U.S. Department of Labor's Medical Care Consumer Price Index. ${ }^{56}$

\section{CHOICE OF MODEL}

A probabilistic cost-utility microsimulation state-transition model was developed. We chose microsimulation rather than a cohort model, as it is particularly suited to chronic diseases. It allows for the incorporation of heterogeneity and the tracking of events, the mapping of long periods of time while taking into consideration disease progression and varying probabilities.

The model began after failure of the patients' first TNFi. Patients then passed through sequences of up to 3 biological drugs (adalimumababatacept-tocilizumab for the cycling arm or abatacept-tocilizumab-rituximab for switching to a non-TNFi arm), after which they shifted to csDMARDs (Figure 1). 


\section{FIGURE 1 Health State Transition Diagram}

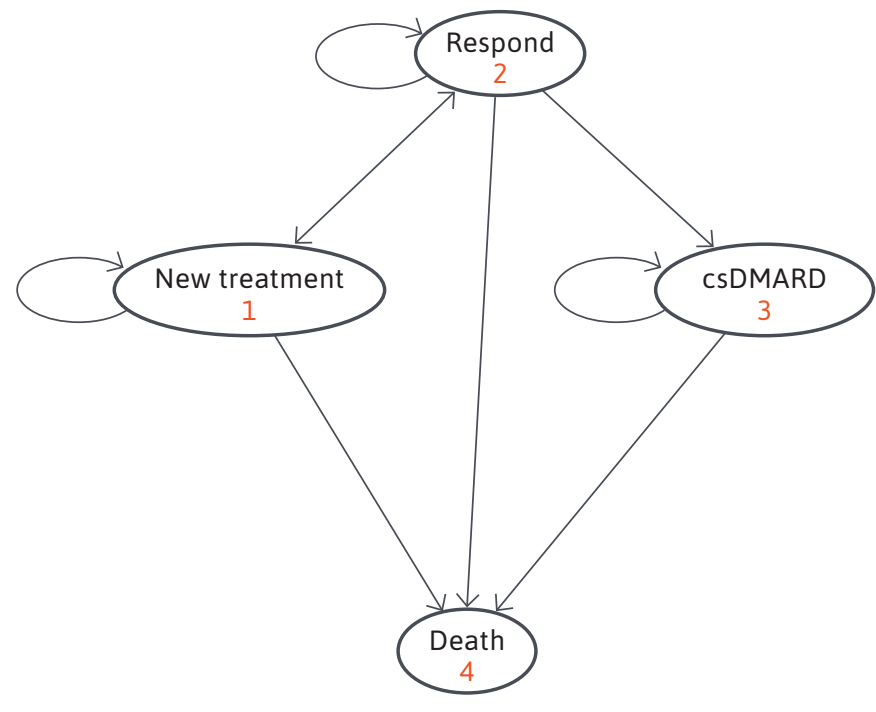

Note: Arrows indicate shifts among health states, and arrows on the arcs represent the direction of the possible movements.

$\operatorname{cs} D M A R D=$ conventional synthetic disease-modifying antirheumatic drugs.

\section{ASSUMPTIONS}

In the current analysis, we assumed that there was an immediate loss of treatment effect after discontinuation. ${ }^{17}$ This is based on the expectation that the withdrawal is due to loss of effect or adverse events. Our analysis captured the costs of adverse events indirectly in the calculation of overall health care costs and discontinuation probabilities. ${ }^{57}$ The disutility of adverse events could not be captured with available data.

\section{ANALYTICAL METHODS}

Pairwise comparisons were made between treatment sequences, and the cost-utility evaluated in terms of the incremental cost-effectiveness ratio (ICER). We used a proprietary decision-analytic software (TreeAge Software, Williamstown, MA, version 2019 R2.1). The software's microsimulation summed the utilities and costs of individual patients as they transitioned between health states. It used a Monte Carlo pseudorandom series to generate a new state configuration from the current one. Drug costs differed per treatment, and transition probabilities depended on the treatment as well as the number of cycles.

The first cycle of new treatment was associated with increased costs owing to more intensive initial follow-up and, in some cases, loading doses of the drugs. The first cycle was also potentially associated with the highest utility. Given these circumstances, we did not implement the recommended half-cycle correction. This adjusts the model for differential timing of events but would entail eliminating half of the upfront cost and utility of a new treatment. ${ }^{58}$

Two-dimensional simulation was used to account for both first-order (i.e., variability among individual trials) and second-order (i.e., parameter uncertainty) sampling. We systematically varied the input parameters and probabilities across their possible ranges and calculated the ICERs on the basis of these parameters. For our analysis, we used a \$100,000 per QALY willingness-to-pay threshold. ${ }^{59-63}$

Probabilistic sensitivity analysis was used to assess the joint uncertainty across all parameters. Transition probabilities were assigned beta distributions and costs were assigned gamma distributions. The Monte Carlo simulation then recalculated expected values by repeatedly sampling parameter values from these distributions. By iterating this process 10,000 times, we obtained distributions of the incremental costs and effectiveness for the comparison of the 2 strategies.

\section{Results}

Our model comparing the most common cycling and switching to a non-TNFi strategies after initial TNFi failure showed that the expected cost of switching to a non-TNFi drug was approximately $\$ 263,755$ over 10 years compared with $\$ 252,308$, the expected cost for cycling.

\section{INCREMENTAL COSTS AND OUTCOMES}

The incremental cost of $\$ 11,357$ achieved an expected discounted QALY benefit of 0.16 over those 10 years, for an ICER of $\$ 68,950$ per QALY in the base case (Table 3). We reran the model using different time horizons (5 years and lifetime), alternative baseline HAQ and HAQ-to-utility conversion formulas, and a case in which we did not allow negative QALYs (states worse than death). Varying the utility function resulted in ICERs ranging from \$57,673 per QALY to $\$ 81,233$ per QALY. Modifying baseline HAQ increased the ICER from between $\$ 86,119$ per QALY and \$118,240 per QALY, whereas varying the time horizon resulted in an ICER of $\$ 44,573$ per QALY for the lifetime horizon and $\$ 148,558$ per QALY for the 5-year time horizon. Overall, the median was $\$ 83,676$ per QALY (Table 3), which is within the range of current willingness-to-pay thresholds.

\section{CHARACTERIZING UNCERTAINTY AND HETEROGENEITY}

A cost-effectiveness acceptability curve (Figure 2), used to summarize some of the uncertainty in the analysis, 


\section{TABLE 3 Scenario Analysis Comparing the Cycling Strategy (Adalimumab-Abatacept-Tocilizumab) with the Switching Strategy (Abatacept-Tocilizumab-Rituximab)}

\begin{tabular}{|c|c|c|c|c|c|c|c|}
\hline & \multicolumn{2}{|c|}{ Cycling } & \multicolumn{2}{|c|}{ Switching } & \multirow{2}{*}{$\begin{array}{c}\text { Incremental } \\
\text { Costs, \$ }\end{array}$} & \multirow{2}{*}{$\begin{array}{c}\text { Incremental } \\
\text { QALYs }\end{array}$} & \multirow[b]{2}{*}{ ICER, \$ } \\
\hline & Costs, $\$$ & QALYs & Costs, $\$$ & QALYs & & & \\
\hline Base case & 252,308 & 3.63 & 263,755 & 3.80 & 11,357 & 0.16 & 68,950/QALY \\
\hline Lifetime horizon & 360,538 & 2.73 & 371,935 & 2.98 & 11,397 & 0.26 & 44,573/QALY \\
\hline 5-year horizon & 144,128 & 2.12 & 152,120 & 2.17 & 8,012 & 0.05 & 148,558/QALY \\
\hline Including CCla ${ }^{a}$ & 221,675 & 3.12 & 231,797 & 3.23 & 10,122 & 0.12 & 87,852/QALY \\
\hline Bansback HAQ-QALY & 247,957 & 3.88 & 259,390 & 4.08 & 11,432 & 0.20 & 57,673/QALY \\
\hline Carreño HAQ-QALY & 220,587 & 4.04 & 230,821 & 4.17 & 10,234 & 0.14 & 81,233/QALY \\
\hline No negative QALY & 221,948 & 3.24 & 232,436 & 3.38 & 10,489 & 0.14 & 74,479/QALY \\
\hline ATTAIN trial HAQ & 229,532 & 2.21 & 241,563 & 2.34 & 12,030 & 0.13 & 90,229/QALY \\
\hline REFLEX trial HAQ & 233,589 & 1.98 & 245,619 & 2.12 & 12,030 & 0.14 & 86,119/QALY \\
\hline BRAM HAQ & 240,612 & 1.90 & 253,096 & 2.00 & 12,484 & 0.11 & 118,240/QALY \\
\hline
\end{tabular}

aThirty-three individuals aged $>80$ years at model onset with 2 or more comorbidities.

ATTAIN = Abatacept Trial in Treatment of Anti-TNF Inadequate Responders; BRAM=Birmingham Rheumatoid Arthritis Model; CCla $=$ age-adjusted Charlson Comorbidity Index; $\mathrm{HAQ}=$ Health Assessment Questionnaire; ICER =incremental cost-effectiveness ratio; $Q A L Y=$ quality-adjusted life-years.

demonstrated the probability of cost-effectiveness across a range of willingness-to-pay thresholds given the available data. In this curve, switching to a non-TNFi strategy is more likely to be cost-effective at a willingness-to-pay threshold of just under $\$ 70,000$ per QALY. Below this, the cycling strategy is more likely to be the cost-effective option.

Probabilistic sensitivity analysis suggested that switching to a non-TNFi strategy had an $80.6 \%$ probability of having an ICER below $\$ 100,000$, compared with a 37.1\% probability at the more conservative $\$ 50,000$ per QALY threshold.

In the incremental cost-effectiveness scatterplots, although switching to a non-TNFi strategy could be costeffective at the $\$ 50,000$ per QALY threshold, it was more likely to be so with a higher willingness-to-pay threshold (Supplementary Figure 1, available in online article).

\section{Discussion}

We compared the cost-utility of sequences of therapeutic drugs for rheumatoid arthritis patients cycling to a second TNFi versus switching to a drug with a different mechanism of action after their initial TNFi treatment failed. Our basecase incremental cost-effectiveness ratio was $\$ 68,950$ per QALY and varied from $\$ 44,573$ per QALY to $\$ 148,558$ per QALY in scenario analyses.

Our data indicate that switching to abatacept has an $80.6 \%$ probability of being a cost-effective treatment strategy compared with cycling to adalimumab at a willingness-to-pay threshold of $\$ 100,000$ per QALY. The ICER of switching to a non-TNFi strategy was lower over a longer time period than cycling and was higher in the 5-year time horizon and when baseline HAQ-DI was higher. This can be explained by the greater probability of continuing treatment by switching to a non-TNFi strategy: patients benefit from a lower HAQ-DI at baseline for more time, leading to decreased cumulative costs. However, when HAQ-DI is high to begin with or the time frame is not long enough to accumulate benefits, the higher costs associated with switching to a non-TNFi strategy counter clinical gains. ${ }^{58}$

Our ICER of $\$ 67,483$ per QALY (range: \$40,659/QALY$\$ 129,587 /$ QALY) calibrated well with the BRAM model, which had a higher baseline disability ${ }^{16}$ Their comparison of sequences beginning with abatacept or adalimumab resulted in an ICER $\$ 84,218(95 \%$ credible interval $=\$ 41,928-$ $\$ 275,888 ; 2016$ USD). Few studies have compared cycling with switching to a non-TNFi drug, and those that have used a variety of methods and parameters, resulting in a wide range of ICERs. ${ }^{27,46,47,64-66}$ Only 1 was based on a U.S. population: the model was a decision tree with input parameters derived from randomized controlled trials and the Medicare fee schedule. ${ }^{64}$ Over 2 years, the switching sequence of tofacitinib-abatacept-rituximab dominated the cycling sequence, adalimumab-abatacept-rituximab, in terms of total cost per responder. ${ }^{64}$

To the best of our knowledge, our study is the first full cost-utility analysis investigating cycling to a second TNFi 


\section{FIGURE 2}

\section{Cost-Effectiveness Acceptability Curve Comparing the Cycling Strategy (Adalimumab-Abatacept-Tocilizumab) with the Switching Strategy (Abatacept-Tocilizumab- Rituximab) over a 10-Year Time Horizon}

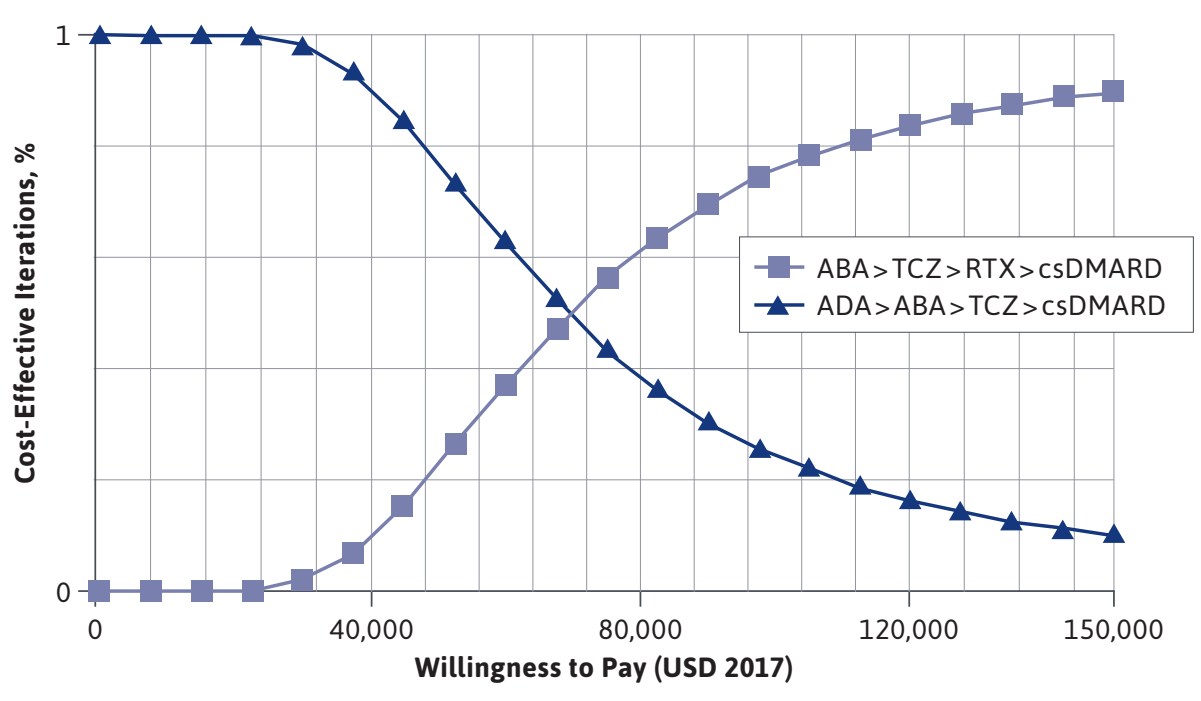

$A B A=$ abatacept $A D A=$ adalimumab; $\operatorname{cs} D M A R D=$ conventional synthetic disease-modifying antirheumatic drugs; $R T X=$ rituximab; $T C Z=$ tocilizumab; $U S D=U . S$. dollar.

(adalimumab) compared with switching to a non-TNFi biological drug (abatacept) after failure of initial TNFi, synthesizing evidence from a U.S. commercial claims database. A review of European cost-utility analyses comparing rituximab or abatacept with TNFi in RA after initial TNFi failure reported ICERs for abatacept ranging from $\$ 86,511$ to more than $\$ 2.2$ million (USD 2016). ${ }^{67}$ Two other reviews concluded that switching to rituximab is a cost-effective alternative to cycling to a second TNFi and may even be cost saving. ${ }^{17,68}$ A recent French study concluded that switching to abatacept after etanercept failure dominated sequences in which patients cycled to adalimumab. ${ }^{32}$ This is likely due both to the different health systems and study methodology that included real-world clinical data and detailed cost parameters.
Compared with the European papers, our lower ICER may partly be attributed to their analyses of rituximab rather than abatacept and also to a baseline mean HAQ-DI that was lower than that of other cost-utility analyses. ${ }^{17}$ This is due to the reliance of other analyses on clinical trial data and, in 1 case, a British cohort. People enrolling in randomized clinical trials tend to have higher disease activity than those in general practice, ${ }^{69,70}$ and the criteria for biologic drugs are stricter in the United Kingdom than in the United States. ${ }^{70}$ Because the primary outcome of our study was incremental effectiveness, this factor likely did not affect the direction of our results.

The greatest strength of our model was its use of real-world data. Treatment sequences were chosen in an objective manner with no implicit preference for a particular outcome. Our earlier systematic review showed that other models were largely funded by industry and, despite abatacept being the most common non-TNFi drug used after initial TNFi failure, most studies investigated rituximab. ${ }^{17}$ An additional advantage of real-world data is the long-term follow-up, which reduces reliance on extrapolation. Clinical trials are limited to $1 \mathrm{drug}$ and are usually conducted over 6 months. Long-term extension studies of up to 2 years do exist but, overall, there is a paucity of head-to-head studies for treatment sequences after initial TNFi. Our use of administrative data also provided a more diverse population than that available from controlled clinical trials. Our costs and discontinuation probabilities were derived from community practice. It has also been reported that using data from controlled trials results in lower ICERs than those from community-based settings. ${ }^{71}$

\section{LIMITATIONS}

Our model had some limitations. We were unable to account for indirect costs, the illness-related productivity losses carried by society as a whole. These are estimated to comprise more than $50 \%$ of total costs attributed to RA but are not available in claims data and cannot be reliably estimated.72

Decision-analysis models are, by definition, simplifications of complex processes and as such cannot capture the full nuance of real-life situations. For example, although population risk stratification is recommended for increased generalizability and application of the model to subgroups (e.g., seropositivity, reason for discontinuation of first TNFi), our lack of individual demographic and clinical data prevented us from adjusting for these variables. Our choice of time horizon may have affected results, therefore, to demonstrate the robustness of the model, we conducted sensitivity analyses using 5-year and lifetime horizons. 
Our model was also limited by the fixed-treatment sequences, which did not account for the fact that the choice of the next drug may depend on the reason for failure of its predecessor (i.e., adverse event or primary versus secondary nonresponse). It is difficult to predict how this would affect results. Also, our results only reflect cycling from TNFi (other than adalimumab) to adalimumab, inferences about cycling from another TNFi may not be robust. Similarly, our choice of switching to a non-TNFi strategy was based on our previously reported claims data in which we did not find much use of JAK-inhibitors. ${ }^{8}$ Future studies will be needed to explore the new emerging treatments that are being approved for treatment of RA patients who have not responded to TNFi as new data of their use becomes available.

The HAQ-DI deterioration rate has been shown to affect study results, and like others, the current study modeled slow, universal HAQ-DI deterioration during therapy. Lack of data on HAQ-DI progression per agent likely affected the accuracy of the model. Similarly, pain has been shown to be an independent predictor of health-related quality of life and should be incorporated into the HAQ-DI to utility conversion formula. ${ }^{73}$ This information is not available from administrative databases but would be a useful addition to future studies.

\section{Conclusions}

To the best of our knowledge, our study is the first full cost-utility analysis investigating cycling to a second TNFi (adalimumab) compared with switching to a non-TNFi biological drug (abatacept) after failure of the initial TNFi, synthesizing evidence from a U.S. commercial claims database. Our results support the existing literature showing that swapping is a cost-effective strategy for patients with RA whose initial TNFi therapy failed at a willingness-to-pay threshold of $\$ 100,000$.

\section{DISCLOSURES}

Funding for this project was provided by a Rheumatology Research Foundation Investigator Award (principal investigator: Maria A. Lopez-Olivo). Karpes Matusevich's work was supported by a Doctoral Dissertation Research Award from the University of Texas, School of Public Health Office of Research. Lal reports competing interests outside of the submitted work (employed by Optum). Suarez-Almazor reports competing interests outside of the submitted work (consulting fees from Pfizer, AbbVie, Eli Lilly, Agile Therapeutics, Amag Pharmaceuticals, and Gilead). Chan, Swint, and Cantor have nothing to disclose.

\section{ACKNOWLEDGMENTS}

This manuscript was part of a PhD dissertation that has been published online (https://digitalcommons.library.tmc.edu/ uthsph dissertsopen/71/) and, as such, much of the text is similar.

\section{REFERENCES}

1. Hresko A, Lin TC, Solomon DH. Medical care costs associated with rheumatoid arthritis in the U.S.: a systematic literature review and meta-analysis. Arthritis Care Res (Hoboken). 2018;70(10):1431-38.

2. Furst DE, Breedveld FC, Kalden JR, et al. Updated consensus statement on biological agents, specifically tumour necrosis factor $\alpha(\mathrm{TNF} \alpha)$ blocking agents and interleukin-1 receptor antagonist (IL-1ra), for the treatment of rheumatic diseases, 2005. Ann Rheum Dis. 2005;64(Suppl 4):iv2-14.

3. Singh JA, Christensen R, Wells GA, et al. Biologics for rheumatoid arthritis: an overview of Cochrane reviews. Cochrane Database Syst Rev. 2009(4):CD007848.

4. Emery P. Optimizing outcomes in patients with rheumatoid arthritis and an inadequate response to anti-TNF treatment. Rheumatology (Oxford). 2012;51 (Suppl 5):v22-30.
5. Strand V, Tundia N, Song Y, Macaulay D, Fuldeore M. Economic burden of patients with inadequate response to targeted immunomodulators for rheumatoid arthritis. J Manag Care Spec Pharm. 2018;24(4):344-52. doi: 10.18553/ jmcp.2018.24.4.344

6. Smolen JS, Landewe RBM, Bijlsma JWJ, et al. EULAR recommendations for the management of rheumatoid arthritis with synthetic and biological disease-modifying antirheumatic drugs: 2019 update. Ann Rheum Dis. 2020;79(6):685-99.

7. Singh JA, Saag KG, Bridges SL, Jr., et al. 2015 American College of Rheumatology Guideline for the Treatment of Rheumatoid Arthritis. Arthritis Rheumatol. 2016;68(1):1-26.

8. Karpes Matusevich AR, Duan Z, Zhao H, et al. Treatment sequences after discontinuing a tumor necrosis factor inhibitor in patients with rheumatoid arthritis: a comparison of cycling versus swapping strategies. Arthritis Care Res (Hoboken). June 17, 2020 [Epub ahead of print]. doi: $10.1002 /$ acr.24358

9. Wells AF, Curtis JR, Betts KA, Douglas K, Du EX, Ganguli A. Systematic literature review and meta-analysis of tumor necrosis factor-alpha experienced rheumatoid arthritis. Clin Ther. 2017;39(8):1680-94.e1682.

10. Studenic P, Smolen JS, Alasti F, Aletaha D. Treatment patterns and retention in an inception cohort of 309 RA patients. Ann Rheum Dis. 2014;73: 73:1159-60.

11. Elkin E, Hamburger MI, Kamath T, et al. Response to biologic disease-modifying anti-rheumatic drugs after discontinuation of anti-tumor necrosis factor alpha agents in rheumatoid arthritis patients. Arthritis Rheum. 2013;65:S212-13.

12. Du Pan SM, Scherer A, Gabay C, Finckh A. Differential drug retention between anti-TNF agents and alternative biological agents after inadequate response to an anti-TNF agent in rheumatoid arthritis patients. Ann Rheum Dis. 2012;71(6):997-99. 
13. Rotar Z, Hocevar A, Rebolj Kodre A, Praprotnik S, Tomsic M, Slovenian R. Retention of the second-line biologic disease-modifying antirheumatic drugs in patients with rheumatoid arthritis failing one tumor necrosis factor alpha inhibitor: data from the BioRx.si registry. Clin Rheumatol. 2015;34(10):1787-93.

14. Favalli EG, Biggioggero M, Marchesoni A, Meroni PL. Survival on treatment with second-line biologic therapy: a cohort study comparing cycling and swap strategies. Rheumatology (Oxford). 2014;53(9):1664-68.

15. Benedict A, Vanness DJ, Roy S, Cifaldi MA. Comparative effectiveness of biologic therapies for treating rheumatoid arthritis (RA) in patients who failed an anti tumor necrosis factor agent: A metaregression analysis. Arthritis Rheum. 2010;62:2266.

16. Gottenberg JE, Brocq O, Perdriger A, et al. Non-TNF-targeted biologic vs a second anti-TNF drug to treat rheumatoid arthritis in patients with insufficient response to a first anti-TNF drug: a randomized clinical trial. JAMA. 2016;316(11):1172-80.

17. Karpes AR, Suarez-Almazor ME, Cantor SB, Lal LS, Swint JM, Lopez-Olivo MA. Systematic review of economic evaluations of cycling versus swapping in patients with rheumatoid arthritis after failure to tumor necrosis factor inhibitors. Arthritis Care Res. 2020;72(3):343-52.

18. Philips Z, Bojke L, Sculpher M, Claxton K, Golder S. Good practice guidelines for decision-analytic modelling in health technology assessment: a review and consolidation of quality assessment. Pharmacoeconomics. 2006;24(4):355-71.

19. Sanders GD, Neumann PJ, Basu A, et al. Recommendations for conduct, methodological practices, and reporting of cost-effectiveness analyses: Second Panel on Cost-Effectiveness in Health and Medicine. JAMA. 2016;316(10):1093-103.

20. Gabriel S, Drummond M, Maetzel A, et al. OMERACT 6 Economics Working Group report: a proposal for a reference case for economic evaluation in rheumatoid arthritis. J Rheumatol. 2003;30(4):886-90.
21. Maetzel A, Tugwell P, Boers M, et al. Economic evaluation of programs or interventions in the management of rheumatoid arthritis: defining a consensus-based reference case. J Rheumatol. 2003;30(4):891-86.

22. Husereau D, Drummond M, Petrou S, et al. Consolidated Health Economic Evaluation Reporting Standards (CHEERS) statement. Value Health. 2013;16(2):e1-5.

23. Nakajima A, Inoue E, Shimizu Y, et al. Presence of comorbidity affects both treatment strategies and outcomes in disease activity, physical function, and quality of life in patients with rheumatoid arthritis. Clin Rheumatol. 2015;34(3):41-449.

24. Radner H, Smolen JS, Aletaha D. Comorbidity affects all domains of physical function and quality of life in patients with rheumatoid arthritis. Rheumatology (Oxford). 2011;50(2):381-88.

25. Radner H, Smolen JS, Aletaha D. Impact of comorbidity on physical function in patients with rheumatoid arthritis. Ann Rheum Dis. 2010;69(3):536-41.

26. Marques WV, Cruz VA, Rego J, Silva NA. The impact of comorbidities on the physical function in patients with rheumatoid arthritis. Rev Bras Reumatol Engl Ed. 2016;56(1):14-21.

27. Malottki K, Barton P, Tsourapas A, et al. Adalimumab, etanercept, infliximab, rituximab and abatacept for the treatment of rheumatoid arthritis after the failure of a tumour necrosis factor inhibitor: a systematic review and economic evaluation. Health Technol Assess. 2011;15(14):1-278.

28. Genovese MC, Becker JC, Schiff M, et al. Abatacept for rheumatoid arthritis refractory to tumor necrosis factor alpha inhibition. N Eng J Med. 2005;353(11):1114-23.

29. Bansback NJ, Regier DA, Ara R, et al. An overview of economic evaluations for drugs used in rheumatoid arthritis: focus on tumour necrosis factor-alpha antagonists. Drugs. 2005;65(4):473-96.
30. Bonafede MM, Curtis JR, McMorrow D, Mahajan P, Chen CI. Treatment effectiveness and treatment patterns among rheumatoid arthritis patients after switching from a tumor necrosis factor inhibitor to another medication. Clinicoecon Outcomes Res. 2016;8:707-15.

31. Harnett J, Wiederkehr D, Gerber R, Gruben D, Koenig A, Bourret J. Real-world evaluation of TNF-inhibitor utilization in rheumatoid arthritis. J Med Econ. 2016;19(2):91-102.

32. Ghabri S, Binard A, Pers YM, Maunoury F, Caro JJ. Economic evaluation of sequences of biological treatments for patients with moderate-to-severe rheumatoid arthritis and inadequate response or intolerance to methotrexate in France. Value Health. 2020;23(4):461-70.

33. Baser O, Ganguli A, Roy S, Xie L, Cifaldi M. Impact of switching from an initial tumor necrosis factor inhibitor on health care resource utilization and costs among patients with rheumatoid arthritis. Clin Ther. 2015;37(7):1454-65.

34. Hyrich KL, Lunt M, Watson KD, Symmons DP, Silman AJ; British Society for Rheumatology Biologics R. Outcomes after switching from one anti-tumor necrosis factor alpha agent to a second anti-tumor necrosis factor alpha agent in patients with rheumatoid arthritis: results from a large UK national cohort study. Arthritis Rheum. 2007;56(1):13-20.

35. Hjardem E, Ostergaard M, Podenphant J, et al. Do rheumatoid arthritis patients in clinical practice benefit from switching from infliximab to a second tumor necrosis factor alpha inhibitor? Ann Rheum Dis. 2007;66(9):1184-89.

36. Markenson JA, Gibofsky A, Palmer WR, et al. Persistence with anti-tumor necrosis factor therapies in patients with rheumatoid arthritis: observations from the RADIUS registry. J Rheumatol. 2011;38(7):1273-81.

37. Hyrich KL, Lunt M, Dixon WG, Watson KD, Symmons DP, Register BSRB. Effects of switching between anti-TNF therapies on HAQ response in patients who do not respond to their first antiTNF drug. Rheumatology (Oxford). 2008;47(7):1000-05. 
38. Virkki LM, Valleala H, Takakubo Y, et al. Outcomes of switching antiTNF drugs in rheumatoid arthritis - a study based on observational data from the Finnish Register of Biological Treatment (ROB-FIN). Clin Rheumatol. 2011;30(11):1447-54.

39. Caporali R, Sarzi-Puttini P, Atzeni F, et al. Switching TNF-alpha antagonists in rheumatoid arthritis: the experience of the LORHEN registry. Autoimmun Rev. 2010;9(6):465-69.

40. Ramiro S, Landewe R, van der Heijde D, Harrison D, Collier D, Michaud K. Discontinuation rates of biologics in patients with rheumatoid arthritis: are TNF inhibitors different from non-TNF inhibitors? RMD Open. 2015;1(1):e000155.

41. Stevenson M, Archer R, Tosh J, et al. Adalimumab, etanercept, infliximab, certolizumab pegol, golimumab, tocilizumab and abatacept for the treatment of rheumatoid arthritis not previously treated with disease-modifying antirheumatic drugs and after the failure of conventional disease-modifying antirheumatic drugs only: systematic review and economic evaluation. Health Tech Assess. 2016;20(35):1-610.

42. Kobelt G, Lindgren P, Singh A, Klareskog L. Cost effectiveness of etanercept (Enbrel) in combination with methotrexate in the treatment of active rheumatoid arthritis based on the TEMPO trial. Ann Rheum Dis. 2005;64(8):1174-79.

43. Vera-Llonch M, Massarotti E, Wolfe F, et al. Cost-effectiveness of abatacept in patients with moderately to severely active rheumatoid arthritis and inadequate response to tumor necrosis factor-alpha antagonists. J Rheumatol. 2008;35(9):1745-53.

44. Arias E, Xu J. United States Life Tables, 2015. Natl Vital Stat Rep. 2018;67(7):1-64.

45. Wolfe F, Mitchell DM, Sibley JT, et al. The mortality of rheumatoid arthritis. Arthritis Rheum. 1994;37(4):481-94.
46. Kielhorn A, Porter D, Diamantopoulos A, Lewis G. UK cost-utility analysis of rituximab in patients with rheumatoid arthritis that failed to respond adequately to a biologic disease-modifying antirheumatic drug. Curr Med Res Opin. 2008;24(9):2639-50.

47. Hallinen TA, Soini EJ, Eklund K, Puolakka K. Cost-utility of different treatment strategies after the failure of tumour necrosis factor inhibitor in rheumatoid arthritis in the Finnish setting. Rheumatology (Oxford). 2010;49(4):767-77.

48. Merkesdal S, Kirchhoff T, Wolka D, Ladinek G, Kielhorn A, Rubbert-Roth A. Cost-effectiveness analysis of rituximab treatment in patients in Germany with rheumatoid arthritis after etanercept-failure. Eur J Health Econ. 2010;11(1):95-104.

49. Kobelt G, Eberhardt K, Jonsson L, Jonsson B. Economic consequences of the progression of rheumatoid arthritis in Sweden. Arthritis Rheum. 1999;42(2):347-56.

50. Bansback N, Ara R, Karnon J, Anis A. Economic evaluations in rheumatoid arthritis: a critical review of measures used to define health states. Pharmacoeconomics. 2008;26(5):395-408.

51. Bansback NJ, Brennan A, Ghatnekar O. Cost effectiveness of adalimumab in the treatment of patients with moderate to severe rheumatoid arthritis in Sweden. Ann Rheum Dis. 2005;64(7):995-1002.

52. Carreño A, Fernandez I, Badia X, Varela C, Roset M. Using HAQ-DI to estimate HUI-3 and EQ-5D utility values for patients with rheumatoid arthritis in Spain. Value Health. 2011;14(1):192-200.

53. Wailoo AJ, Bansback N, Brennan A, Michaud K, Nixon RM, Wolfe F. Biologic drugs for rheumatoid arthritis in the Medicare program: a cost-effectiveness analysis. Arthritis Rheum. 2008;58(4):939-46.

54. Ohinmaa AE, Thanh NX, Barnabe C, et al. Canadian estimates of health care utilization costs for rheumatoid arthritis patients with and without therapy with biologic agents. Arthritis Care Res. 2014;66(9):1319-27.
55. Klimes J, Vocelka M, Sedova L, et al. Medical and productivity costs of rheumatoid arthritis in the Czech Republic: cost-of-illness study based on disease severity. Value Health Reg Issues. 2014;4:75-81.

56. U.S. Bureau of Labor. U.S. Bureau of Labor's Medical Consumer Price Index 2017. Accessed December 13, 2020. https://www.bls.gov/data/

57. Heather EM, Payne K, Harrison M, Symmons DP. Including adverse drug events in economic evaluations of antitumour necrosis factor-alpha drugs for adult rheumatoid arthritis: a systematic review of economic decision analytic models. Pharmacoeconomics. 2014;32(2):109-34.

58. Sonnenberg FA, Beck JR. Markov models in medical decision making: a practical guide. Med Decis Making. 1993;13(4):322-38.

59. Neumann PJ, Cohen JT, Weinstein MC. Updating cost-effectiveness-the curious resilience of the $\$ 50,000-$ per-QALY threshold. N Eng J Med. 2014;371(9):796-97.

60. Neumann PJ, Cohen JT. QALYs in 2018-advantages and concerns. JAMA. 2018;319(24):2473-74.

61. Sanders GD, Maciejewski ML, Basu A. Overview of cost-effectiveness analysis. JAMA. 2019;321(14):1400-01.

62. Weinstein MC. How much are Americans willing to pay for a quality-adjusted life year? Med Care. 2008;46(4):343-345.

63. Willke RJ, Neumann PJ, Garrison LP, Jr., Ramsey SD. Review of recent U.S. value frameworks-a health economics approach: an ISPOR Special Task Force Report [6]. Value Health. 2018;21(2):155-60.

64. Claxton L, Jenks M, Taylor M, et al. An economic evaluation of tofacitinib treatment in rheumatoid arthritis: modeling the cost of treatment strategies in the United States. J Manag Care Spec Pharm. 2016;22(9):1088-102. doi: 10.18553/ jmcp.2016.22.9.1088 
65. Manders SHM, Kievit W, Adang E, et al. Cost-effectiveness of abatacept, rituximab, and TNFi treatment after previous failure with TNFi treatment in rheumatoid arthritis: a pragmatic multi-centre randomised trial. Arthritis Res Ther. 2015;17:134.

66. Lindgren P, Geborek P, Kobelt G. Modeling the cost-effectiveness of treatment of rheumatoid arthritis with rituximab using registry data from Southern Sweden. Int J Technol Assess Health Care. 2009;25(2):181-89.

67. Joensuu JT, Huoponen S, Aaltonen KJ, Konttinen YT, Nordstrom D, Blom M. The cost-effectiveness of biologics for the treatment of rheumatoid arthritis: a systematic review. PLoS One. 2015;10(3):e0119683.
68. Sullivan SD, Alfonso-Cristancho R, Carlson J, Mallya U, Ringold S. Economic consequences of sequencing biologics in rheumatoid arthritis: a systematic review. J Med Econ. 2013;16(3):391-96.

69. Monti S, Grosso V, Todoerti M, Caporali R. Randomized controlled trials and real-world data: differences and similarities to untangle literature data. Rheumatology (Oxford). 2018;57 (57 Suppl 7):vii54-58.

70. Kremer JM. Time for change in the conduct of clinical trials in patients with rheumatoid arthritis. J Rheumatol. 2011;38(10):2087-88.
71. Farahani P, Levine M, Goeree R. A comparison between integrating clinical practice setting and randomized controlled trial setting into economic evaluation models of therapeutics. J Eval Clin Pract. 2006;12(4):463-70.

72. Batko B, Rolska-Wojcik P, Wladysiuk M. Indirect costs of rheumatoid arthritis depending on type of treatment: a systematic literature review. Int J Environ Res Public Health. 2019;16(16):2966.

73. Pennington B, Davis S. Mapping from the Health Assessment Questionnaire to the EQ-5D: the impact of different algorithms on cost-effectiveness results. Value Health. 2014;17(8):762-71. 